\title{
Spatial Quantum Correlations in Multiple Scattered Light
}

\author{
P. Lodahl, ${ }^{*}$ A. P. Mosk, and A. Lagendijk ${ }^{\dagger}$ \\ Complex Photonic Systems, Department of Science and Technology and MESA ${ }^{+}$Research Institute, University of Twente, \\ P.O. Box 217, 7500 AE Enschede, The Netherlands
}

(Received 4 February 2005; published 19 October 2005)

\begin{abstract}
We predict a new spatial quantum correlation in light propagating through a multiple scattering random medium. The correlation depends on the quantum state of the light illuminating the medium, is infinite in range, and dominates over classical mesoscopic intensity correlations. The spatial quantum correlation is revealed in the quantum fluctuations of the total transmission or reflection through the sample and should be readily observable experimentally.
\end{abstract}

DOI: 10.1103/PhysRevLett.95.173901

PACS numbers: 42.25.Dd, 42.50.Lc, 78.67. $-\mathrm{n}$

When light propagates through a disordered scattering medium, a strongly modulated interference structure, known as a speckle pattern, is generated. Speckle patterns are the most direct manifestation of wave coherence in transport of light through samples that are thicker than the transport mean free path $\ell$, which is the average distance over which the direction of light is diffused due to random scattering. By analyzing the statistical properties, such volume speckle patterns reveal strong correlations that are responsible for fundamental physical phenomena such as the memory effect $[1,2]$ and enhanced mesoscopic fluctuations [3-7]. Furthermore, clear signatures of Anderson localization of light have been observed by analyzing intensity fluctuations [8].

While spatial correlations of the intensity of multiply scattered light have been investigated intensely, spatial correlations hidden in the quantum fluctuations of light have to our knowledge not been addressed. Here we predict that strong quantum correlations exist between spatially separated parts of a far-field speckle pattern that have no classical analog. The quantum correlation is infinite range, since it is independent of the angular separation between the parts of the speckle pattern under investigation, and dominates over mesoscopic fluctuations. Therefore, speckle patterns generated with quantum light are much stronger correlated than classical theory predicts, and the quantum corrections should be observable even for a moderate amount of scattering. In contrast, the magnitude of classical intensity correlations are all found to scale with the inverse of the mesoscopic conductance $g=N \ell / L$, where $N$ is the number of conducting modes and $L$ is the thickness of the scattering medium [3-7]. Only for extremely strong scattering, i.e., close to the Anderson localization transition $(g \simeq 1)$, do such mesoscopic correlations dominate.

The quantum correlation will be shown to depend on the quantum state of light illuminating the multiple scattering sample. The physical origin of the effect is due to distribution of photon correlations of the input state over the spatial degrees of freedom of the speckle pattern. This mechanism was previously predicted to lead to new long- range correlations of thermal radiation from a disordered waveguide [9]. We evaluate the correlation function for three different quantum states: coherent state, thermal state, and Fock state. For a coherent state the spatial correlation vanishes, while strong correlations and anticorrelations are found for the thermal state and the Fock state, respectively. We show that the spatial quantum correlation can be measured conveniently by recording the quantum noise of the total transmission or reflection from the medium. Such measurements were carried out very recently for a coherent state [10] and appear feasible also in the near future for other quantum states. Our work is connected to the field of quantum imaging, where spatial correlations are employed for reconstructing images [11].

We describe the propagation of quantum states of light through a nonabsorbing, multiple scattering medium using the formalism developed by Beenakker et al. $[12,13]$. The model describes effectively a quasi-1D configuration, but this approximation is known to be excellent also for a 3D slab geometry. The annihilation operator $\hat{a}_{b}$ for an output mode $b$ is coupled to the annihilation operators associated with all input modes $\hat{a}_{a^{\prime}}^{\text {in }}$ and $\hat{a}_{b^{\prime}}^{\text {in }}$ through the relation $\hat{a}_{b}=$ $\sum_{a^{\prime}} t_{a^{\prime} b} \hat{a}_{a^{\prime}}^{\text {in }}+\sum_{b^{\prime}} r_{b^{\prime} b} \hat{a}_{b^{\prime}}^{\text {in }}$, where $t$ and $r$ are electric field transmission and reflection coefficients, respectively, and the summations are over all $N$ input and output modes. We use the notation that $a$ denotes modes to the left and $b$ modes to the right of the medium; cf. Fig. 1. The modes correspond to different propagation directions ( $k$ vectors) of the incoming and outgoing light as measured directly in the far field.

In the following, we explicitly outline the calculation of the quantum fluctuations of the light transmitted through the sample when light is coupled through a single input mode $a$. In the quantum description, vacuum fluctuations coupled into all other input modes $a^{\prime} \neq a$ and $b^{\prime}$ must be included. The operator $\hat{n}_{b}=\hat{a}_{b}^{\dagger} \hat{a}_{b}$ describes the number of photons transmitted to the output mode $b$, and the total number of transmitted photons is obtained by adding all output modes: $\hat{n}_{T}=\sum_{b} \hat{n}_{b}$. In an experiment, the total transmission can be measured with an integrating sphere cf. Fig. 1. The quantum fluctuations are quantified through 


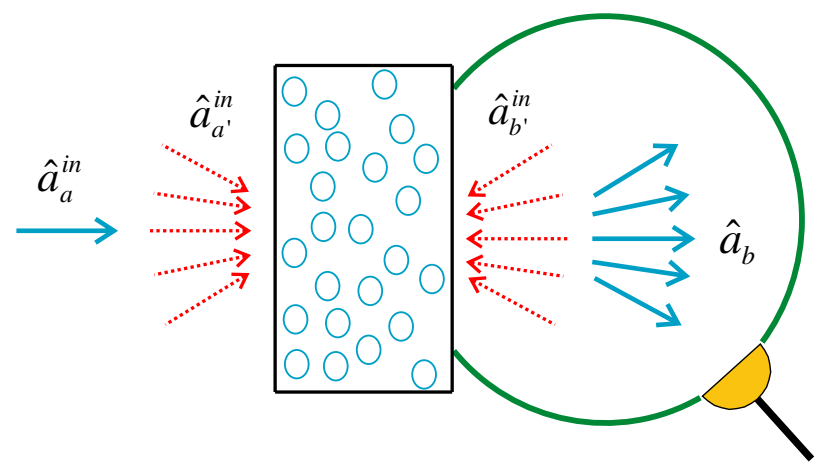

FIG. 1 (color online). Total transmission through a multiple scattering sample. A quantum state of light is coupled through mode $a$ and the total number of photons is collected with an integrating sphere. Vacuum fluctuations in all open channels $a^{\prime} \neq a$ and $b^{\prime}$ must be included in the quantum description.

the variance of the photon number: $\Delta n^{2} \equiv\left\langle\hat{n}^{2}\right\rangle-\langle\hat{n}\rangle^{2}$, where $\langle\cdot\rangle$ is the quantum mechanical expectation value. The fluctuations of the total transmission are straightforwardly found to be

$$
\Delta n_{T}^{2}=\sum_{b} \Delta n_{b}^{2}+\sum_{b_{0}} \sum_{b_{1} \neq b_{0}}\left\langle\hat{n}_{b_{0}} \hat{n}_{b_{1}}\right\rangle-\left\langle\hat{n}_{b_{0}}\right\rangle\left\langle\hat{n}_{b_{1}}\right\rangle,
$$

where an index specifying the fixed input mode $(a)$ has been omitted for brevity of notation. We immediately see that the variance of the total transmission is different from the sum of the variances of all outgoing modes. Also cross correlations between different output modes contribute, and these correlations are shown to be significant in the following.

In order to evaluate Eq. (1), $\left\langle\hat{n}_{b}\right\rangle$ and $\left\langle\hat{n}_{b_{0}} \hat{n}_{b_{1}}\right\rangle$ must be computed. The nonvanishing contributions are

$$
\begin{aligned}
\left\langle\hat{n}_{b}\right\rangle= & \sum_{a^{\prime}, a^{\prime \prime}} t_{a^{\prime} b}^{*} t_{a^{\prime \prime} b}\left\langle\hat{a}_{a^{\prime}}^{\text {in }} \hat{a}_{a^{\prime \prime}}^{\text {in }}\right\rangle, \\
\left\langle\hat{n}_{b_{0}} \hat{n}_{b_{1}}\right\rangle= & \sum_{a^{\prime}, a^{\prime \prime}, a^{\prime \prime \prime}, a^{\prime \prime \prime \prime}} t_{a^{\prime} b_{0}}^{*} t_{a^{\prime \prime} b_{0}} t_{a^{\prime \prime \prime} b_{1}}^{*} t_{a^{\prime \prime \prime \prime} b_{1}}\left\langle\hat{a}_{a^{\prime}}^{\text {in } \dagger} \hat{a}_{a^{\prime \prime}}^{\text {in }} \hat{a}_{a^{\prime \prime \prime}}^{\text {in }} \hat{a}_{a^{\prime \prime \prime}}^{\text {in }}\right\rangle \\
& +\sum_{a^{\prime}, b^{\prime}, b^{\prime \prime}, a^{\prime \prime}} t_{a^{\prime} b_{0}}^{*} r_{b^{\prime} b_{0}} r_{b^{\prime \prime} b_{1}}^{*} t_{a^{\prime \prime} b_{1}}\left\langle\hat{a}_{a^{\prime}}^{\text {in } \dagger} \hat{a}_{b^{\prime}}^{\text {in }} \hat{a}_{b^{\prime \prime}}^{\text {in } \dagger} \hat{a}_{a^{\prime \prime}}^{\text {in }}\right\rangle .
\end{aligned}
$$

The expectation values of the various operator products can be evaluated using that the vacuum is coupled through all other channels than $a$. We obtain

$$
\begin{aligned}
& \left\langle\hat{a}_{a^{\prime}}^{\text {in }} \hat{a}_{a^{\prime \prime}}^{\text {in }}\right\rangle=\left\langle\hat{n}_{a}^{\text {in }}\right\rangle \delta_{a^{\prime}, a} \delta_{a^{\prime \prime}, a}, \\
& \left\langle\hat{a}_{a^{\prime}}^{\text {in }} \hat{a}_{b^{\prime}}^{\text {in }} \hat{a}_{b^{\prime \prime}}^{\text {int }} \hat{a}_{a^{\prime \prime}}^{\text {in }}\right\rangle=\left\langle\hat{n}_{a}^{\text {in }}\right\rangle \delta_{a^{\prime}, a} \delta_{a^{\prime \prime}, a} \delta_{b^{\prime}, b^{\prime \prime}}, \\
& \left\langle\hat{a}_{a^{\prime}}^{\text {in }} \hat{a}_{a^{\prime \prime}}^{\text {in }} \hat{a}_{a^{\prime \prime \prime}}^{\text {in }} \hat{a}_{a^{\prime \prime \prime}}^{\text {in }}\right\rangle=\delta_{a^{\prime}, a} \delta_{a^{\prime \prime \prime \prime}, a}\left[\left\langle\hat{n}_{a}^{\text {in }}\right\rangle \delta_{a^{\prime \prime}, a^{\prime \prime \prime}}+\left(\left\langle\hat{n}_{a}^{\text {in } 2}\right\rangle\right.\right. \\
& \left.\left.-\left\langle\hat{n}_{a}^{\mathrm{in}}\right\rangle\right) \delta_{a^{\prime \prime}, a} \delta_{a^{\prime \prime \prime}, a}\right] \text {. }
\end{aligned}
$$

By combining Eqs. (2) and (3) using an identity derived from commutation relations [14], the photon number variance of mode $b$ and the cross correlation between two different directions $b_{0} \neq b_{1}$ can be found

$$
\begin{aligned}
\frac{\overline{\Delta n_{b}^{2}}}{\left\langle\hat{n}_{a}^{\text {in }}\right\rangle} & =\overline{T_{a b}}+{\overline{T_{a b}}}^{2}\left(F_{a}^{\text {in }}-1\right)(2+8 / 3 g), \\
\frac{\overline{\left\langle\hat{n}_{b_{0}} \hat{n}_{b_{1}}\right\rangle}-\overline{\left\langle\hat{n}_{b_{0}}\right\rangle\left\langle\hat{n}_{b_{1}}\right\rangle}}{\left\langle\hat{n}_{a}^{\text {in }}\right\rangle} & ={\overline{T_{a b}}}^{2}\left(F_{a}^{\text {in }}-1\right)(1+4 / 3 g) .
\end{aligned}
$$

Here we have introduced the intensity transmission coefficients $T_{a b}=\left|t_{a b}\right|^{2}$ that have been averaged over all realizations of disorder (denoted by a bar). Products of transmission coefficients are expanded to first order in $1 / g$, i.e., $\overline{T_{a b_{0}} T_{a b_{1}}} \approx\left(1+\delta_{b_{0}, b_{1}}\right)(1+4 / 3 g){\overline{T_{a b}}}^{2} \quad$ [15]. The Fano factor $F_{a}^{\text {in }}=\Delta\left(n_{a}^{\text {in }}\right)^{2} /\left\langle\hat{n}_{a}^{\text {in }}\right\rangle$ measures the variance of the photon number relative to the average number of photons and is equal to unity for Poissonian photon statistics. We evaluate the Fano factor for three different singlemode quantum states of light: a coherent state (CS), a thermal state (TS), and an $n$-photon Fock state (FS), which correspond to $\left(F_{a}^{\mathrm{in}}\right)_{\mathrm{CS}}=1, \quad\left(F_{a}^{\mathrm{in}}\right)_{\mathrm{TS}}=1+\left\langle\hat{n}_{a}^{\mathrm{in}}\right\rangle$, and $\left(F_{a}^{\mathrm{in}}\right)_{\mathrm{FS}}=0$, respectively [16].

The classical transmission coefficient can be calculated from the theory of light diffusion, which leads to $\overline{T_{a b}}=$ $\ell / N L$ in the absence of absorption [17]. Inserting Eqs. (4) in Eq. (1), we arrive at the final expression for the quantum fluctuations of the total transmission

$$
\frac{\overline{\Delta n_{T}^{2}}}{\left\langle\hat{n}_{a}^{\text {in }}\right\rangle}=\frac{\ell}{L}+\frac{\ell^{2}}{L^{2}}\left(F_{a}^{\text {in }}-1\right)\left[1+\frac{1}{g}\left(\frac{4}{3}+\frac{\ell}{L}\right)\right] .
$$

Neglecting at present correction terms of order $1 / g$, the variance of the total transmission is seen to contain a linear and a quadratic term in $\ell / L$. The former is the sum of the variances of each individual channel, which corresponds to incoherently adding the fluctuations of each output mode. The quadratic term is due to correlations between different output modes. Note that, while a quadratic scaling was found also for transmission of classical noise through a multiple scattering medium [10], the contribution discussed here is a quantum effect. The fluctuations of the total reflection can be calculated in a similar way, and neglecting correction terms of order $1 / \mathrm{g}$ leads to

$$
\frac{\overline{\Delta n_{R}^{2}}}{\left\langle\hat{n}_{a}^{\text {in }}\right\rangle}=\left(1-\frac{\ell}{L}\right)+\left(1-\frac{\ell}{L}\right)^{2}\left(F_{a}^{\text {in }}-1\right) .
$$

Figure 2 displays the variance of the total transmission and reflection for three different quantum states of light as a function of the ratio of the mean free path $\ell$ to the sample length $L$. For a coherent state, the variance scales linearly, which was experimentally confirmed in Ref. [10]. For other quantum states of light, a quadratic term contributes by either a positive (for the thermal state) or a negative amount (for the Fock state), which means that the photon statistics of the total transmission and reflection is super-Poissonian or sub-Poissonian, respectively. These quantum correlations are found to be most pronounced in 


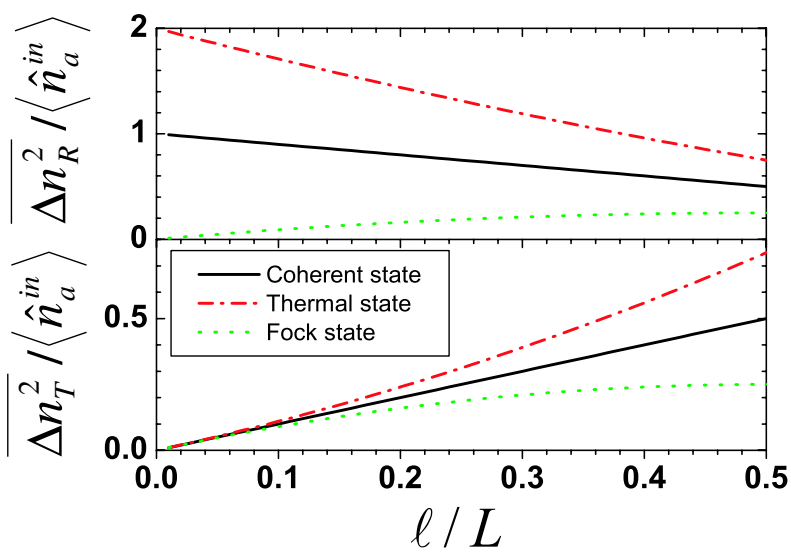

FIG. 2 (color online). Variance of the quantum fluctuations of the total reflection (upper plot) and total transmission (lower plot) for a coherent state (solid line), a thermal state with $\left\langle\hat{n}_{a}^{\text {in }}\right\rangle=1$ (dashed-dotted line), and an arbitrary Fock state (dotted line) as a function of $\ell / L$. All correction terms of order $1 / g$ have been neglected. In the reflection (transmission), pronounced variations in the photon statistics are found for decreasing (increasing) $\ell / L$.

the reflection for thick samples $(\ell / L \rightarrow 0)$ and in the transmission for thin samples $(\ell / L \rightarrow 1)$. This behavior can be understood intuitively, since for thick (thin) samples relatively few photons are transmitted (reflected) and vacuum fluctuations coupled through all open modes lead to Poissonian photon statistics. The validity of the diffusion model for light propagation requires $\ell / L \lesssim 1$, and in this limit each transmitted or reflected photon has experienced numerous scattering events. Remarkably, the quantum correlations of these multiply scattered photons are preserved, and, for $\ell / L \rightarrow 0$, the fluctuations of the total reflection equal the fluctuations of the input light. This clearly demonstrates that, deep in the multiple scattering regime, the quantum corrections are most pronounced in the reflection and, thereby, devises a route for experimental observation of quantum correlations in a volume speckle pattern.

The quadratic terms in Eqs. (5) and (6) originate from spatial correlations between different output directions in the speckle pattern. We define the two-point spatial correlation function, which is evaluated using Eqs. (2) and (3) and [14]

$$
C_{b_{0} b_{1}} \equiv \frac{\overline{\left\langle\hat{n}_{b_{0}} \hat{n}_{b_{1}}\right\rangle}}{\left\langle\hat{n}_{b_{0}}\right\rangle\left\langle\hat{n}_{b_{1}}\right\rangle}=1+\frac{F_{a}^{\text {in }}-1}{\left\langle\hat{n}_{a}^{\text {in }}\right\rangle},
$$

where $b_{0} \neq b_{1}$. $C_{b_{0} b_{1}}$ gauges the correlation between photons in modes $b_{0}$ and $b_{1}$ [18] and depends only on the statistics of the input light. Note that Eq. (7) is exact and independent of $1 / \mathrm{g}$. The correlation function discussed here is fundamentally different from the one encountered in simpler systems as, e.g., a dual-port beam splitter, since Eq. (7) is averaged over all ensembles of disorder. To measure the two-point correlation function generally requires involved coincidence detection schemes between different output directions from the medium. However, we find here that the quantum fluctuations of the total transmission, or, equivalently, the total reflection, provide an alternative way of extracting the two-point correlation. The total transmission and the quantum correlation function are connected through

$$
\frac{\overline{\Delta n_{T}^{2}}}{\left\langle\hat{n}_{a}^{\mathrm{in}}\right\rangle}=\frac{\ell}{L}+\frac{\ell^{2}}{L^{2}}\left\langle\hat{n}_{a}^{\mathrm{in}}\right\rangle\left[C_{b_{0} b_{1}}-1\right],
$$

where again corrections of order $1 / g$ have been neglected.

Figure 3 shows the two-point correlation function for three different quantum states when varying the average number of photons in the input state. For a coherent state with an arbitrary average number of photons the correlation function is unity, i.e., the spatial correlations vanish. For single-mode thermal states, the spatial correlation function is always equal to 2, which means that different parts of the speckle pattern are strongly correlated. For Fock states, a striking nonclassical behavior is found: The spatial correlation function is reduced below unity, which means that the spatial directions are anticorrelated. This corresponds to spatial antibunching of light, and the correlation function $C_{b_{0} b_{1}}$ is the spatial counterpart of the second-order coherence function $g^{(2)}(\tau)$ that is omnipresent in quantum optics [19]. For a single-photon Fock state, the correlation function vanishes identically, since, if a photon is detected in one channel, the probability of detecting another photon in a different channel is zero. For Fock states with an increasing number of photons, the correlation function approaches unity, i.e., the spatial correlations vanish.

In addition to the spatial correlations, mesoscopic fluctuations also contribute to the quantum fluctuations of the

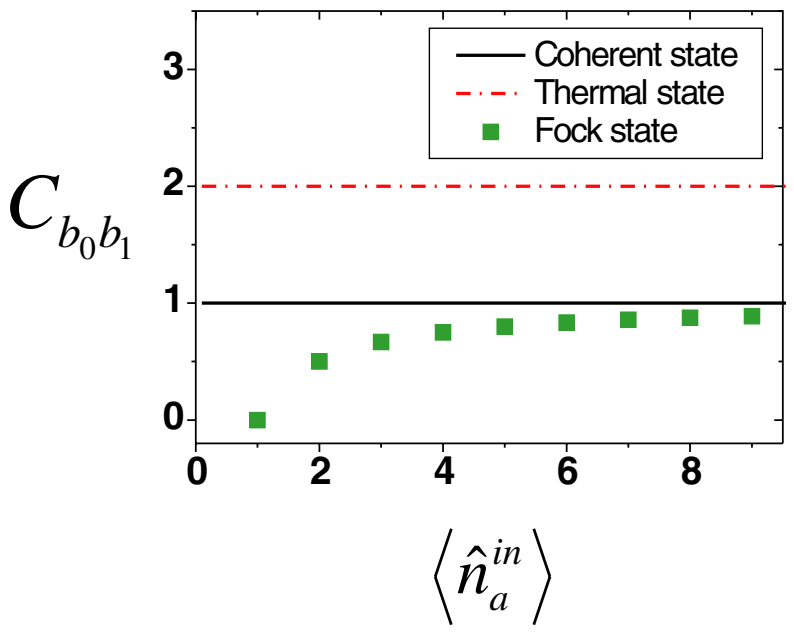

FIG. 3 (color online). Two-point correlation function $C_{b_{0} b_{1}}$ for three different quantum states of light as a function of the average number of photons in the incoming field. The correlation function exhibits either strong correlation (thermal state) or anticorrelation (Fock state) relative to the uncorrelated case (coherent state). 


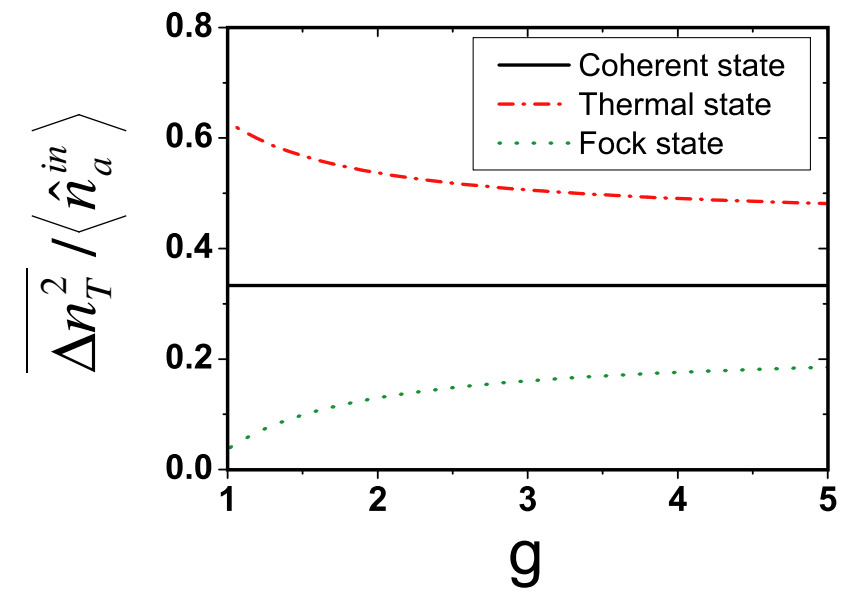

FIG. 4 (color online). Fluctuations of the total transmission for a coherent state, a thermal state with $\left\langle\hat{n}_{a}^{\text {in }}\right\rangle=1$, and an arbitrary Fock state as a function of the mesoscopic conductance $g$ and for a fixed amount of disorder: $\ell / L=1 / 3$ For decreasing $g$, the mesoscopic correlations either enhance or suppress quantum fluctuations for the thermal state and the Fock state, respectively. The fluctuations of the coherent state are unaltered for all values of $g$. When $g$ approaches unity, the localization regime is entered and the model is invalid.

total transmission, cf. Eq. (5). Figure 4 displays these fluctuations as a function of the mesoscopic conductance $g$. For small values of $g$, the mesoscopic correlations either enhance or reduce the quantum fluctuations for thermal states and Fock states, respectively. In contrast, the quantum fluctuations for a coherent state of light are unaltered. These mesoscopic fluctuations provide another example of correlations that are present only in a quantum description of multiple scattering.

It is instructive to compare our findings on photon fluctuations in diffusive propagation to the transport of electrons through an elastically scattering disordered conductor. In the diffusive regime of electron transport, Poissonian electron fluctuations are reduced, which is a direct consequence of the Fermi-Dirac statistics of electrons in each individual conduction channel [20,21]. However, no correlations exist between different conduction channels and the fluctuations can simply be added up incoherently. Our work demonstrates that the dominating correlation in diffusive transport of photons is due to correlation between different spatial modes that are the optical analogy to electronic conduction channels, thus pointing out a fundamental difference between electrons and photons.

We have predicted a spatial correlation present in a quantum description of multiple scattering of light. The spatial correlation depends on the quantum state of the light source illuminating the sample and can be measured by recording the quantum fluctuations of the total transmission or reflection from the sample. Our work shows that multiple scattering of nonclassical light induces stronger correlations than for classical light. The predictions should be readily observable experimentally even for moderate amounts of scattering.

This work is part of the research program of the "Stichting voor Fundamenteel Onderzoek der Materie (FOM)," which is financially supported by the "Nederlandse Organisatie voor Wetenschappelijk Onderzoek (NWO)."

*Present address: Research Center COM, Technical University of Denmark, Dk-2800 Lyngby, Denmark.

Electronic address: pel@com.dtu.dk

${ }^{\dagger}$ Present address: FOM Institute for Atomic and Molecular Physics (AMOLF), P.O. Box 41883, 1009 DB Amsterdam, The Netherlands.

[1] S. Feng, C. Kane, P. A. Lee, and A. D. Stone, Phys. Rev. Lett. 61, 834 (1988).

[2] I. Freund, M. Rosenbluh, and S. Feng, Phys. Rev. Lett. 61, 2328 (1988).

[3] M. P. van Albada, J.F. de Boer, and A. Lagendijk, Phys. Rev. Lett. 64, 2787 (1990).

[4] F. Scheffold and G. Maret, Phys. Rev. Lett. 81, 5800 (1998).

[5] B. Shapiro, Phys. Rev. Lett. 83, 4733 (1999).

[6] A. Garcia-Martin, F. Scheffold, M. Nieto-Vesperinas, and J. J. Saenz, Phys. Rev. Lett. 88, 143901 (2002).

[7] S. E. Skipetrov, Phys. Rev. Lett. 93, 233901 (2004).

[8] A. A. Chabanov, M. Stoytchev, and A.Z. Genack, Nature (London) 404, 850 (2000).

[9] M. Patra and C. W. J. Beenakker, Phys. Rev. A 59, R43 (1999).

[10] P. Lodahl and A. Lagendijk, Phys. Rev. Lett. 94, 153905 (2005).

[11] L. A. Lugiato, A. Gatti, and E. Brambilla, J. Opt. B 4, S176 (2002)

[12] C. W. J. Beenakker, Phys. Rev. Lett. 81, 1829 (1998).

[13] M. Patra and C. W. J. Beenakker, Phys. Rev. A 61, 063805 (2000).

[14] As a direct consequence of the commutation relation for the output modes, we have the identity $\left[\hat{a}_{b_{0}}, \hat{a}_{b_{1}}^{\dagger}\right]=$ $\sum_{a^{\prime}} t_{a^{\prime} b_{0}} t_{a^{\prime} b_{1}}^{*}+\sum_{b^{\prime}} r_{b^{\prime} b_{0}} r_{b^{\prime} b_{1}}^{*}=\delta_{b_{0}, b_{1}}$.

[15] R. Berkovits and S. Feng, Phys. Rep. 238, 135 (1994).

[16] L. Mandel and E. Wolf, Optical Coherence and Quantum Optics (Cambridge University Press, New York, 1995).

[17] M.C. W. van Rossum and Th. M. Nieuwenhuizen, Rev. Mod. Phys. 71, 313 (1999).

[18] We note that the correlation function $C_{b_{0} b_{1}}$ defined here is different from the classical correlation function of relevance for intensity measurement, as given, e.g., in Ref. [15].

[19] R. Loudon, The Quantum Theory of Light (Oxford University Press, New York, 2000).

[20] C. W. J. Beenakker and M. Büttiker, Phys. Rev. B 46, R1889 (1992).

[21] Ya. M. Blanter and M. Büttiker, Phys. Rep. 336, 1 (2000). 